Vol. 3, No. 2 | July - December 2020

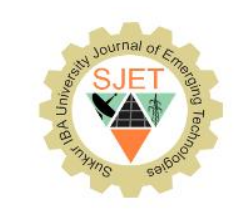

\title{
A Global System for Mobile Communication based Resource Allocation technique to control Autonomous Robotic Glove for Spinal Cord Implant paralysed Patients using Flex Sensors
}

\author{
Hamayun Khan ${ }^{1,2}$, Muhammad Yousaf Ali khan ${ }^{1}$, Irfan uddin ${ }^{2}$, Muhammad \\ Usman Hashmi ${ }^{2}$, Junaid Iqbal ${ }^{3}$, Muhammad Shaheer ${ }^{1}$, Muhammad \\ Zunnurain ${ }^{1}$, Muhammad Umar ${ }^{1}$
}

\begin{abstract}
:
The autonomous robotic glove is designed for paralysed patients, so they would be able to control several electrical appliances around them as well as send an alert message to a particular person. Developing countries are trying to abate the problems of these patients as they depend on others to control nearby appliances both in home and in hospital. In this research we proposed a solution by development $\&$ implementation of robotic glove. The research implementation consists of two parts. First part is transmitter and second part is receiver. Transmitter part mainly consists of flex sensors, arduino uno and HC-11. Flex sensors are used to convert finger movements into different voltage levels, while Arduino is used to read this information from flex sensors and convey it to arduino mega on receiving end as programed by predefined gestures. HC-11 is a serial wireless communication transceiver module which is used to transmit information from Arduino uno to arduino mega on receiving side. Receiver side consist of HC11, arduino mega, Global system for mobile communication (GSM) module, Optocoupler and Triac. Arduino-Mega is used to read the received information and perform action accordingly. On this side we have different electrical appliances which are controlled by hand gestures of patients as well as GSM module which is used to send different messages to associated person. Opto-coupler is used to isolate low power receiving circuit from high power load circuit. Triac is used for switching of AC supply. It is portable, requires low operating power on a single lithium-ion rechargeable battery and having less weight thus user friendly and affordable.
\end{abstract}

Keywords: Flex Sensors, HC-11 module, Arduino, Opto-Coupler, GSM module

\section{Introduction:}

In Pakistan usually, the paralysed patients are not aware from the modern technologies and they feel helpless in front of others. Several Developing countries are trying to overcome the problems of these patients by creating and developing some kind of tools or methods through which paralysed patient can communicate with others and could live a comfort life as other healthy persons[1]. Difficulties around the paralysed patient is increasing day by day usually patient find it difficult to control electrical appliances both in home and hospital and required another person help to either switch on or off the electrical appliances. Paralysed patient face

\footnotetext{
${ }^{1}$ Department of Electrical Engineering, Gomal University, D.I.Khan, KPK, Pakistan

${ }^{2}$ Department of Computer Science, Superior University Lahore, Pakistan

3 Department of Quaid-e-Awam University Larkana-Campus, Pakista
} 
Hamayun Khan (et al.) A Global System for Mobile Communication based Resource Allocation technique to control Autonomous Robotic Glove for Spinal Cord Implant Paralysed Patients using Flex Sensors

(pp. $13-23)$

many difficulties just for daily routine work for example eating food, going to washroom, turn on and off electrical loads etc. To reduce their dependency on others we design a system which would help those patients and allow them to control their daily activities on their own [2]. This research the design of an inimitable product which helps those individuals who are suffering from the disease of paralysis and cannot manage their everyday activities, they are generally known as stroke patients. Mostly these patients are sent home where they live a dependent life. We have designed and implemented a research which could help the paralysed patients to manage their everyday chores to some extent without being reliant on others [3]. This research shows the plan, execution, and assessment of smart framework for stroke patient. The framework, a glove, uses segments of motion acknowledgment resolving the issues of cost, nosiness, and precision while giving a structure to augmentations to the framework. The framework is assessed as far as its energy utilization to evaluate the adequacy and feasibility of a device. So we named it ARGFSCIP (Autonomous Robotic Glove for Spinal Cord Implant patients). There is no doubt every paralysed patient wants to live the life as healthy person. How many times we interact with the paralysed person or a person who cannot stay on your own feet, basically this life just offers a little room for a paralysed patient who cannot move and control anything. One way to boost the mentality and recovery of these patients is to encourage them by positive attitude. Motivation is the tool which doesn't let the paralysed patient to lose hope. It is the best tool and medicine for a paralysed patient who is suffering from such boring life so we wanted to design a research that can comfort the life of stroke or paralysed patient $[4,5]$.

Paralyse or stroke patient can enormously decrease the self-rule and personal satisfaction of a patient while introducing a noteworthy repeating cost at home and $h$ care centers. Improve the system with a non-intrusive wearable sensor framework; the patient can recover a level of self-governance at a small amount of the cost of home medical caretakers $[6,7]$.

\section{Literature review}

Usually paralysed patient find it difficult to control electrical appliances both in home and in hospital and require another help of person to either switch on/off the relevant devices. Development \& implementation of robotic glove to overcome the difficulty of paralysed patient which help them to live an independent and comfortable life Robotic glove is designed for the Patients who lost the mobility in their hands as well as in proper body due to the spinal cord injury so these patients rely on the caregiver to perform his basic task. Paralysed patient required at least one person for support. The proposed research make it easy, we uses gestures reading technique to control the home appliances like fan, bulb, emergency bell and alert doctor by sending massage in case of emergency. So this robotic glove reduces the difficulties of paralysed patients and gives hope, patients, confidence, motivation and independence. Robotic glove is a wearable hand rehabilitation solution for the paralysed patients there are many techniques which are used in the past for this purpose, some of them are Speaking Gloves for Speechless Persons [1]. Hand Gestures Detection and Recognition Building System for Stroke Patients using Supervised Neural Networks A flex sensor or bend sensor is a sensor that measures the amount of deflection or bending. Usually, the sensor is stuck to the surface, and resistance of sensor element is varied by bending the surface. [2]. Recent Developments of Robotic Exoskeletons for Hand Rehabilitation. [3]. Plan and Implementation of the Advanced Wireless Tongue Drive [4]. Sign language interpreter using a robotic glove [5].

Development of Robotic glove system for therapy treatment. [6] IMU sensor based electronic goniometric glove for clinical finger development [7]. Robotic glove for Sign Language communications [8]. robotic glove with motion acknowledgment capacity for the hearing and discourse weakened [9]. Hand Gesture Recognition for Physically Disabled People [10,11] 


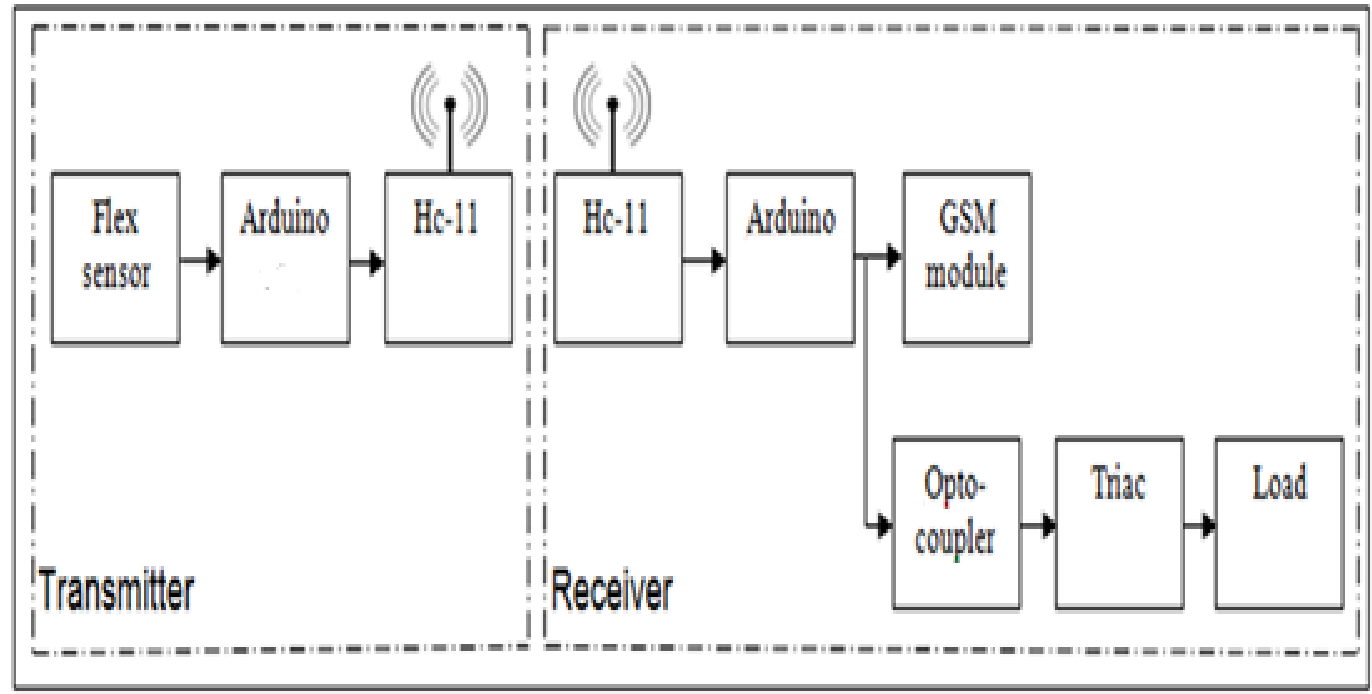

Fig 1: Flex Sensor Communication with GSM module

A Low-Cost Robotic glove for Hand Functions Evaluation [12]. Helping-Hand: A Data Glove Technology for Rehabilitation of Monoplegia Patients [13]. Low Cost robotic glove for remote control by the physically challenged. Towards a battery-free wireless robotic glove for rehabilitation applications based on RFID [14].

\section{Methodology}

This Proposed research Method consists of 2 parts. 1st part is transmitter and 2nd part is receiver. Transmitter part consists of robotic glove controller which consist of flex Sensors, Arduino uno and HC-11. Flex sensor is used to read the finger movement while Arduino is used to program the glove to predefined gesture and read the information from flex sensor. $\mathrm{HC} 11$ is a serial wireless communication transceiver module. HC 11 module is used for transmit an information which is get by Arduino uno from flex sensor.

\section{Flow Chart}

The flow chart in fig 2 tells that how this autonomous robotic glove can perform function, when user twist its finger as an input then this user finger's output in form of digital will go to Arduino uno. Arduino uno recognize the gesture if predefined gesture is not recognize then it will return to start otherwise it will transmit signal to arduino mega through HC-11 which act as transmitter and receiver. Arduino mega will perform two functions either it will send the message or turn on/off the load.

\section{Experimental Results}

We obtained the output of flex sensor by using the analogue to digital converter. After this we relate these analogues to digital value to voltages then we check the output voltage of flex sensor at an angle zero degree (flat) and ninety degree (bend). We calculate reading for five fingers separately because variation for each finger is different.

An Analog to Digital Converter (ADC) is an exceptionally helpful element that changes analog values to digital form. By changing over from the simple world to the computerized world, we can start to utilize hardware to interface to the analog world around us.

The ADC on the Arduino is a 10-bit ADC meaning it can distinguish $1,024\left(2^{\wedge} 10\right)$ discrete simple levels. 
Hamayun Khan (et al.) A Global System for Mobile Communication based Resource Allocation technique to control

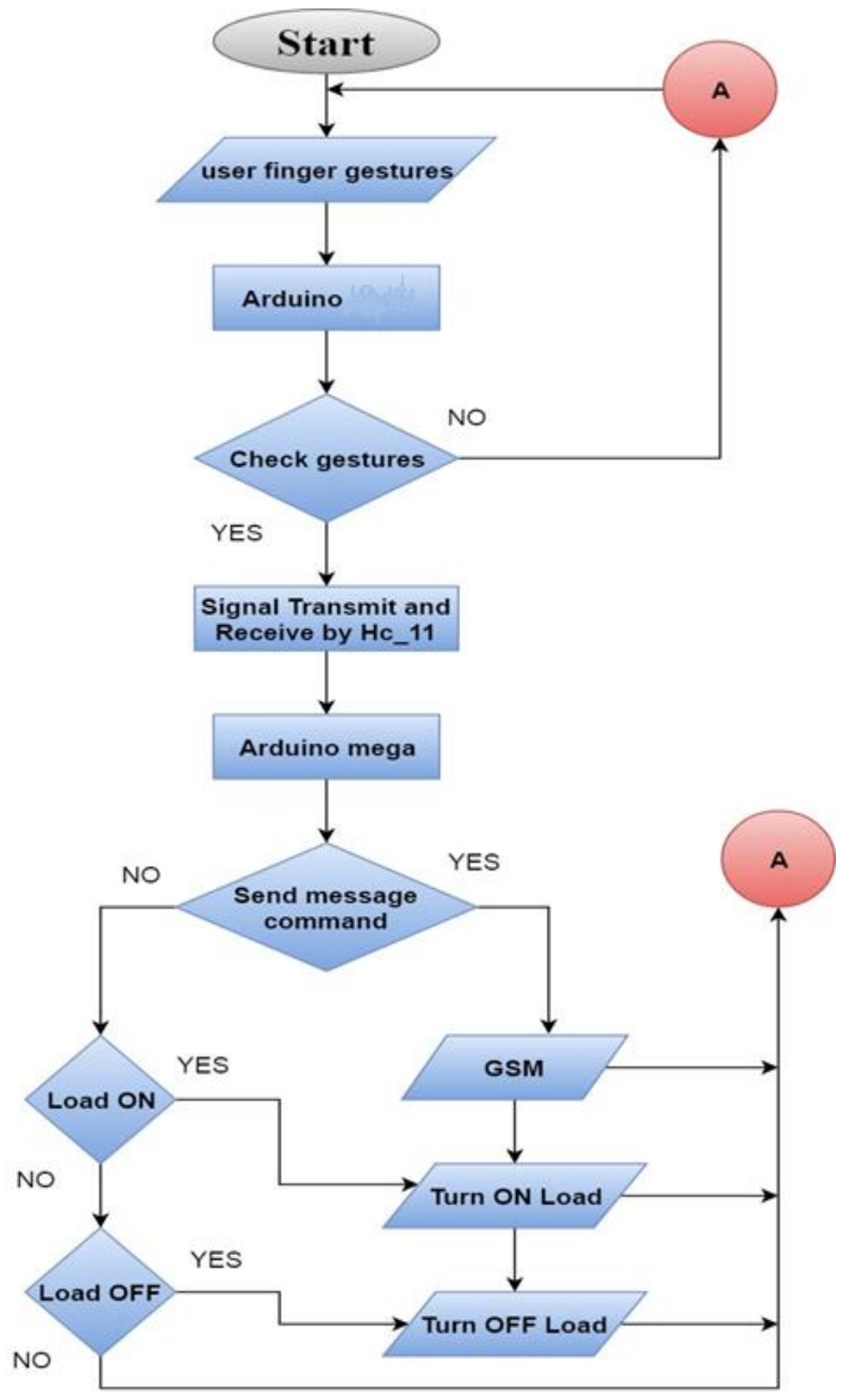

Fig 2: Flow Chart 
Hamayun Khan (et al.) A Global System for Mobile Communication based Resource Allocation technique to control Autonomous Robotic Glove for Spinal Cord Implant Paralysed Patients using Flex Sensors

Voltage $=\frac{\text { System Voltage }}{\text { Resolution ofADC }} *$ ADC Reading $\quad \ldots . . .$.

\section{For Little Finger}

At angle $0^{\circ}$ :

Voltage $=\frac{3}{1023} * 893=2.61 \mathrm{~V}$

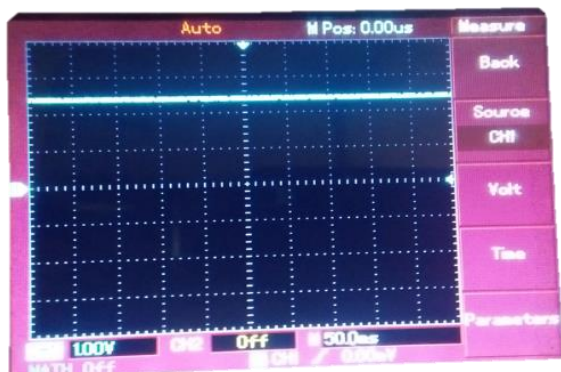

Fig 3: Little Finger Flat Resistance Scope View

In Figure 3 along $\mathrm{x}$-axix time is represented and along y-axix voltage is represented.

One block along $\mathrm{x}$-axix represents $1 \mathrm{v}$ and along y-axix one block shows 50ms time period.

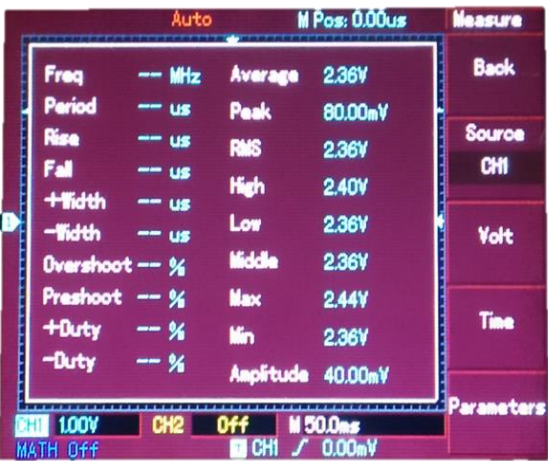

Fig 4: Flat Resistance Parameters

Figure 4 represents the voltage output at 0 degree angle and we took the average value of voltage which is $2.36 \mathrm{v}$ for little finger.

\section{At angle $90^{\circ}$ :}

Voltage $=\frac{3}{1023} * 615=1.80 \mathrm{~V}$

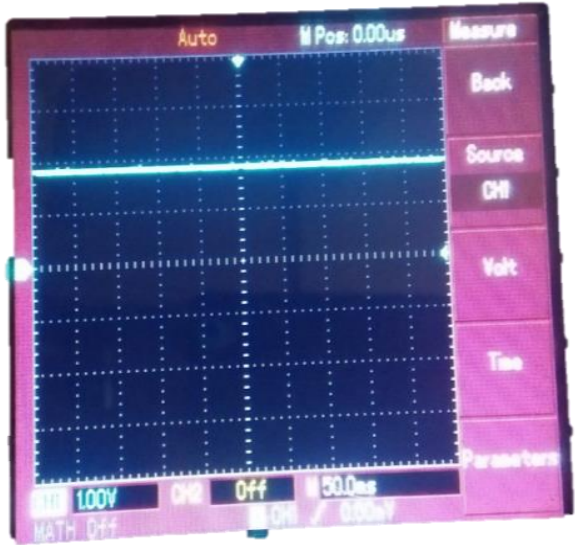

Fig 5: Little Finger Bent Resistance Scope View

Figure 5 along $\mathrm{x}$-axix time is represented and along $y$-axix voltage is represented.

One block along $\mathrm{x}$-axix represents $1 \mathrm{v}$ and along y-axix one block shows 50ms time period.

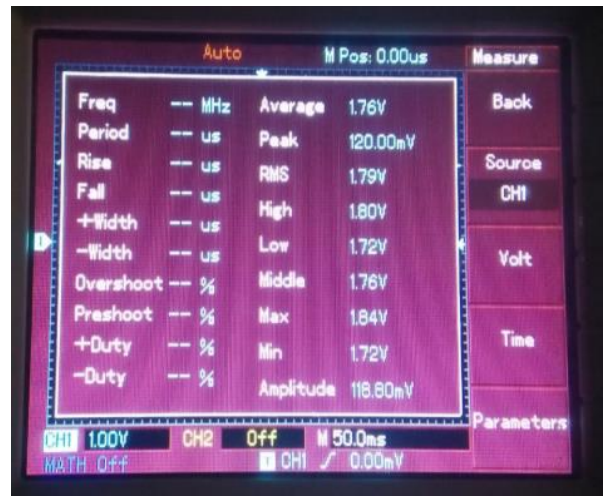

Fig 6:Bent Resistance Parameters

Figure 6 represents the voltage output at 85-degree angle and we took the average value of voltage which is $1.76 \mathrm{v}$ for little finger. 
Hamayun Khan (et al.) A Global System for Mobile Communication based Resource Allocation technique to control Autonomous Robotic Glove for Spinal Cord Implant Paralysed Patients using Flex Sensors (pp. $13-23)$

\section{For Ring Finger}

At angle $0{ }^{\circ}$ :

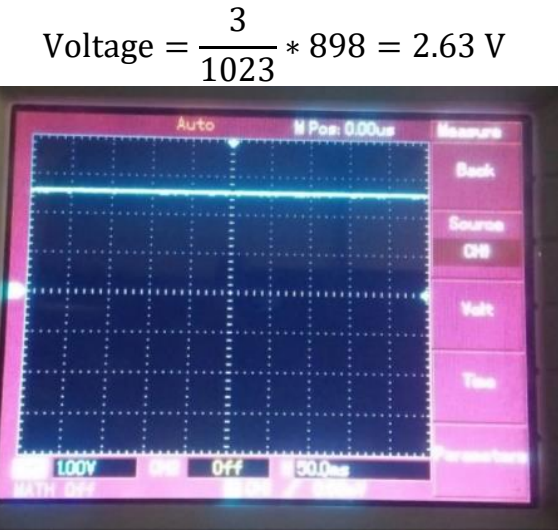

Fig 7: Ring Finger Flat Resistance Scope View

Figure 7 along $\mathrm{x}$-axix time is represented and along y-axix voltage is represented.

One block along $\mathrm{x}$-axix represents $1 \mathrm{v}$ and along y-axix one block shows $50 \mathrm{~ms}$ time period.

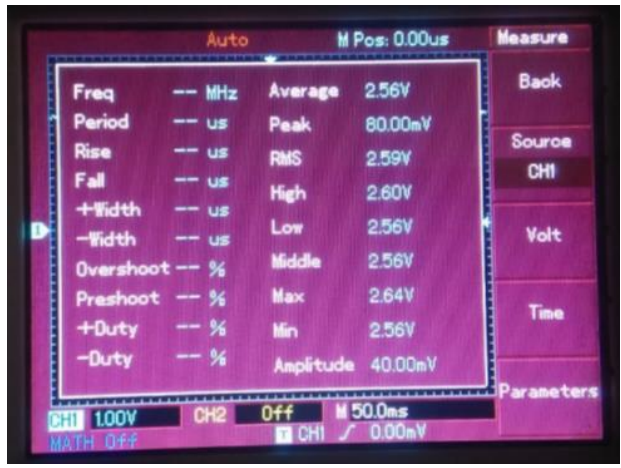

Fig 8: Ring Finger Flat Resistance Scope View

Figure 8 represents the voltage output at 0 degree angle and we took the average value of voltage which is $2.56 \mathrm{v}$ for ring finger.

\section{At angle $90^{\circ}$}

Voltage $=\frac{3}{1023} * 665=1.95 \mathrm{~V}$

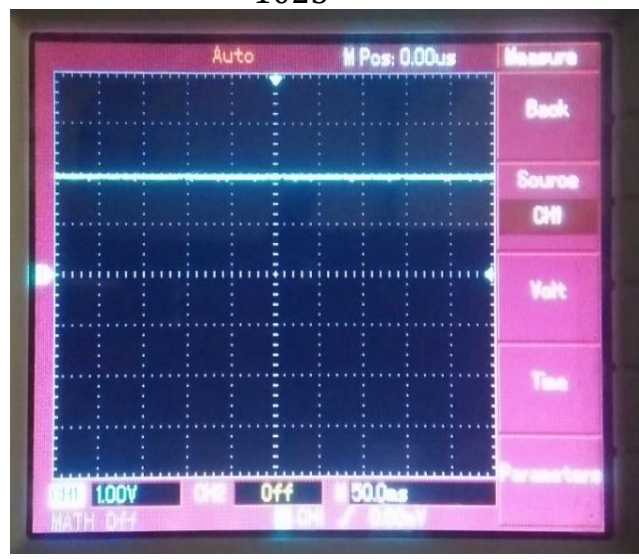

Fig 9: Ring Finger Bent Resistance Scope View

Figure 9 along $\mathrm{x}$-axix time is represented and along $y$-axix voltage is represented.

One block along $\mathrm{x}$-axix represents $1 \mathrm{v}$ and along y-axix one block shows $50 \mathrm{~ms}$ time period.

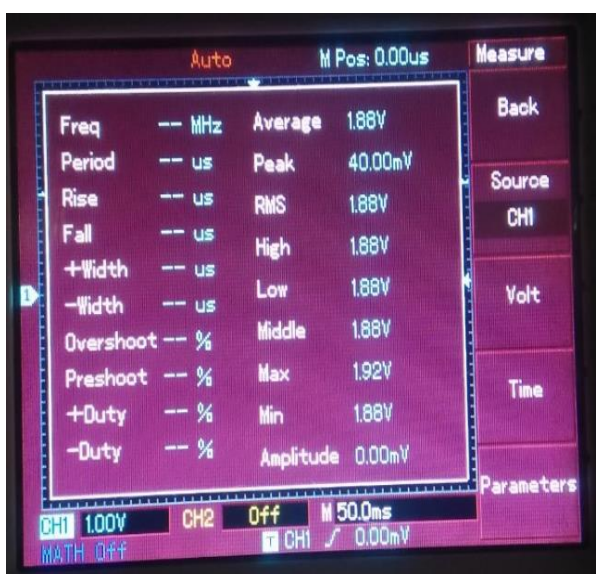

Fig 10: Ring Finger Bent Resistance Parameters

Figure 10 represents the voltage output at 90-degree angle and we took the average value of voltage which is $1.88 \mathrm{v}$ for little finger. 
Hamayun Khan (et al.) A Global System for Mobile Communication based Resource Allocation technique to control Autonomous Robotic Glove for Spinal Cord Implant Paralysed Patients using Flex Sensors (pp. $13-23)$

For Middle Finger

At angle $0^{\circ}$ :

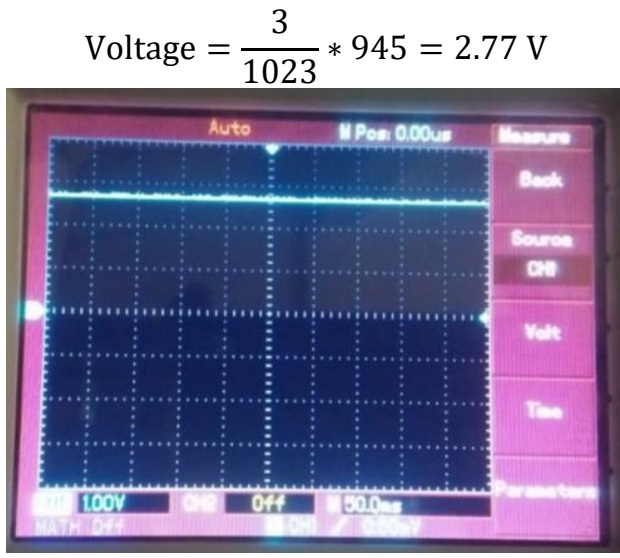

Fig 11: Middle Finger Flat Resistance Scope View

Figure 11 along $\mathrm{x}$-axix time is represented and along y-axix voltage is represented.

One block along $\mathrm{x}$-axix represents $1 \mathrm{v}$ and along y-axix one block shows $50 \mathrm{~ms}$ time period.

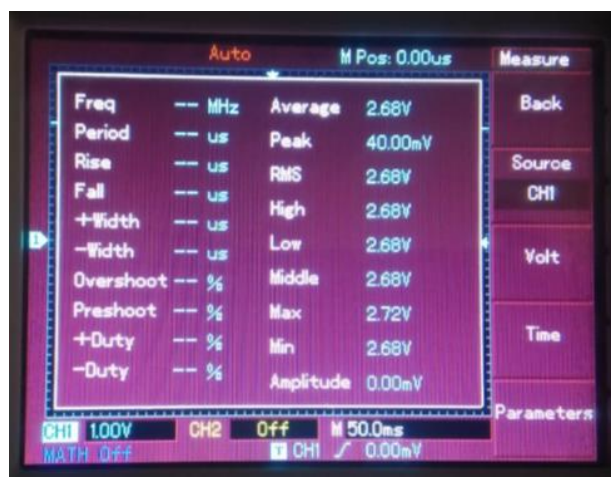

Fig 12: Middle Finger Flat Resistance Parameters

Figure 12 represents the voltage output at 0 -degree angle and we took the average value of voltage which is $2.66 \mathrm{v}$ for middle finger.

\section{At angle $90^{\circ}$ :}

Voltage $=\frac{3}{1023} * 650=1.90 \mathrm{~V}$

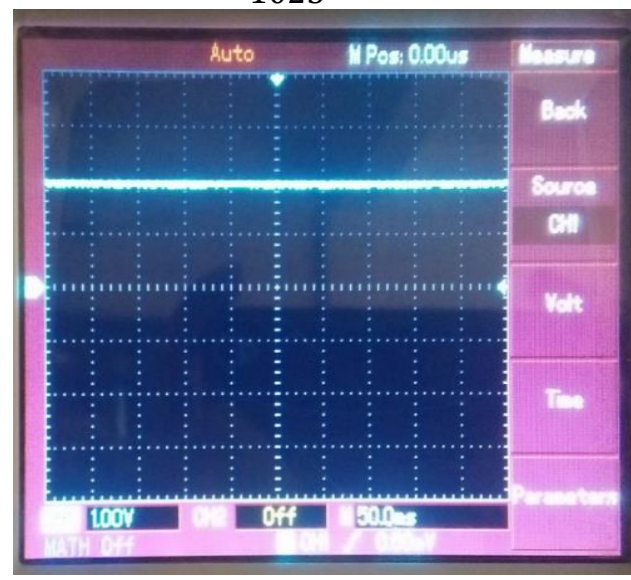

Fig 13: Middle Finger Bent Resistance Scope View

Figure 13 along $x$-axix time is represented and along y-axix voltage is represented.

One block along $\mathrm{x}$-axix represents $1 \mathrm{v}$ and along y-axix one block shows $50 \mathrm{~ms}$ time period.

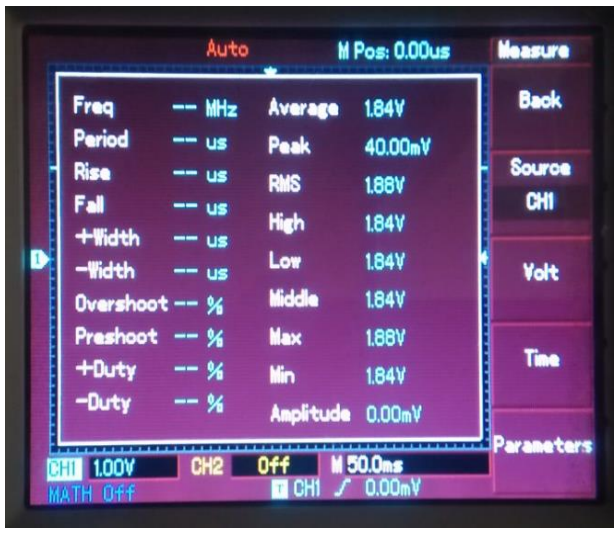

Fig 14: Middle Finger Bent Resistance Parameters

Figure 14 represents the voltage output at 90-degree angle and we took the average value of voltage which is $1.84 \mathrm{v}$ for middle finger. 
Hamayun Khan (et al.) A Global System for Mobile Communication based Resource Allocation technique to control Autonomous Robotic Glove for Spinal Cord Implant Paralysed Patients using Flex Sensors (pp. $13-23)$

\section{For Index Finger}

At angle $0{ }^{\circ}$ :

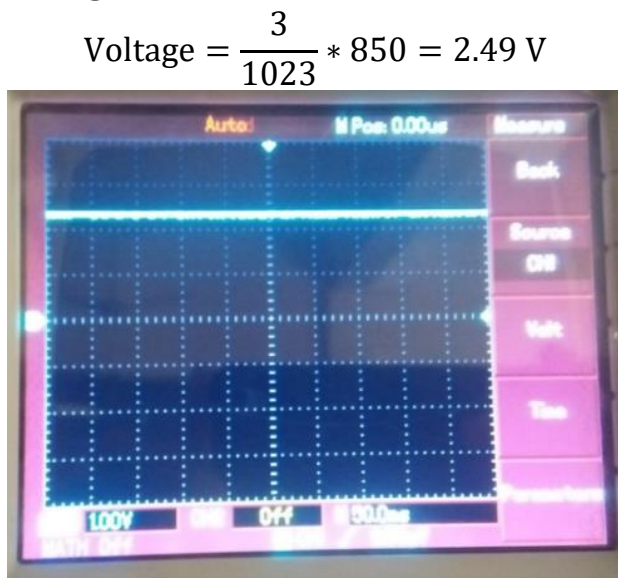

Fig 15: Index inger Flat Resistance Scope View

Figure 15 along $x$-axix time is represented and along y-axix voltage is represented.

One block along $\mathrm{x}$-axix represents $1 \mathrm{v}$ and along y-axix one block shows $50 \mathrm{~ms}$ time period.

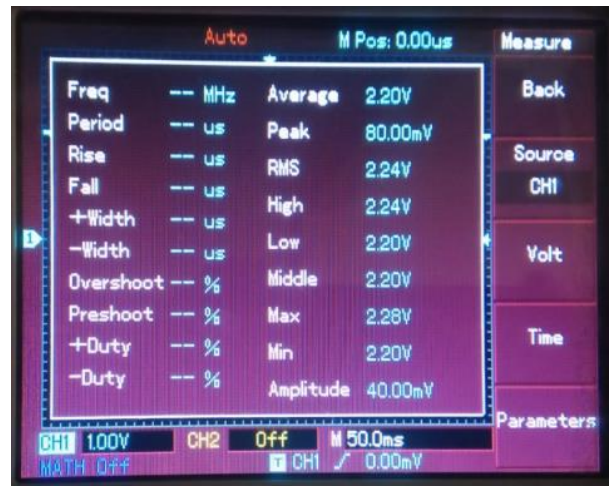

Fig 16: Index Finger Flat Resistance Parameters

Figure 16 represents the voltage output at 0 degree angle and we took the average value of voltage which is 2.20 for index finger.

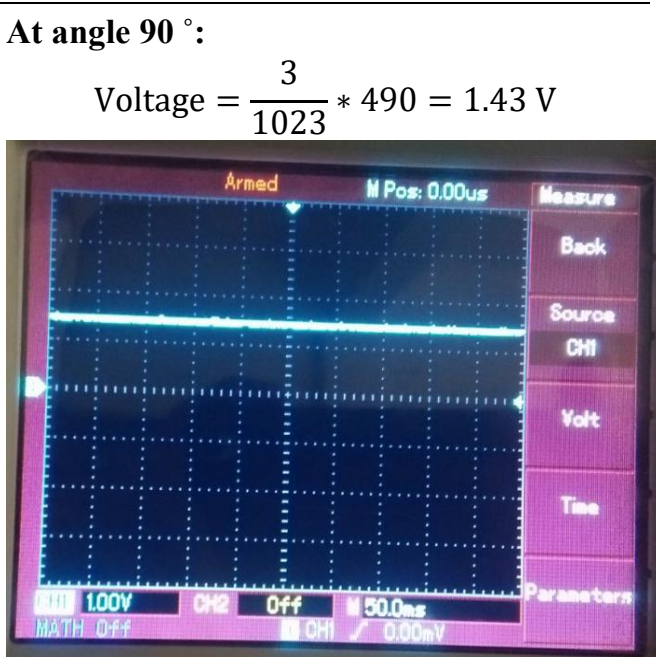

Fig 17: Index Finger Bent Resistance Scope View

Figure 17 along $\mathrm{x}$-axix time is represented and along $\mathrm{y}$-axix voltage is represented.

One block along $\mathrm{x}$-axix represents $1 \mathrm{v}$ and along y-axix one block shows $50 \mathrm{~ms}$ time period.

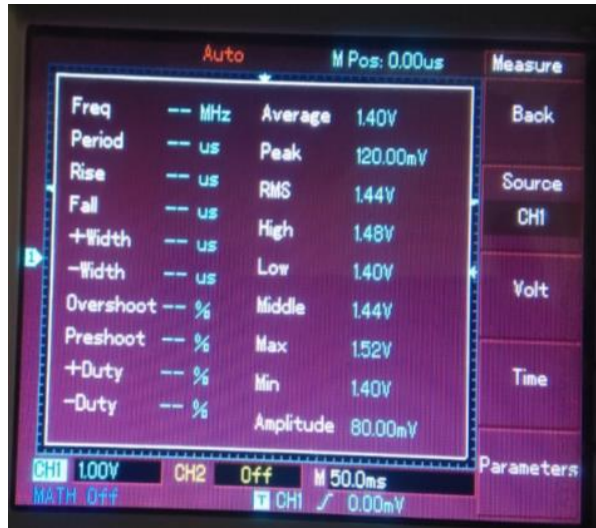

Fig 18: Index Finger Bent Resistance Parameters

Figure 18 represents the voltage output at 90 degree angle and we took the average value of voltage which is $1.40 \mathrm{v}$ for index finger. 
Hamayun Khan (et al.) A Global System for Mobile Communication based Resource Allocation technique to control Autonomous Robotic Glove for Spinal Cord Implant Paralysed Patients using Flex Sensors (pp. $13-23)$

\section{For Thumb}

At angle $0{ }^{\circ}$ :

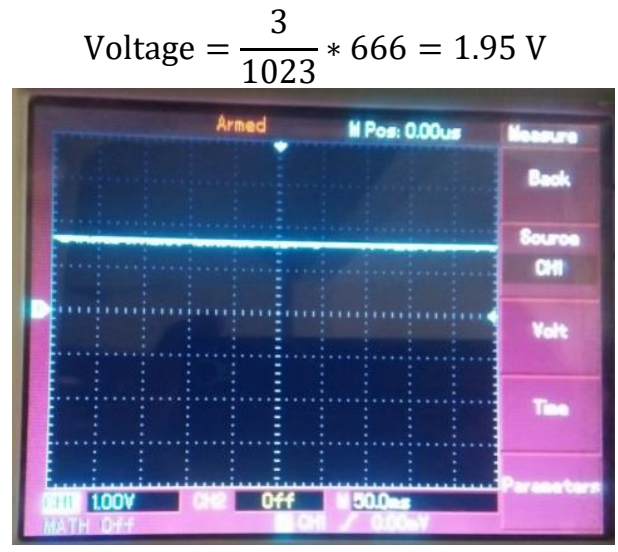

Fig 19: Thumb flat Resistance Scope View

Figure 19 along $\mathrm{x}$-axix time is represented and along y-axix voltage is represented.

One block along $\mathrm{x}$-axix represents $1 \mathrm{v}$ and along y-axix one block shows $50 \mathrm{~ms}$ time period.

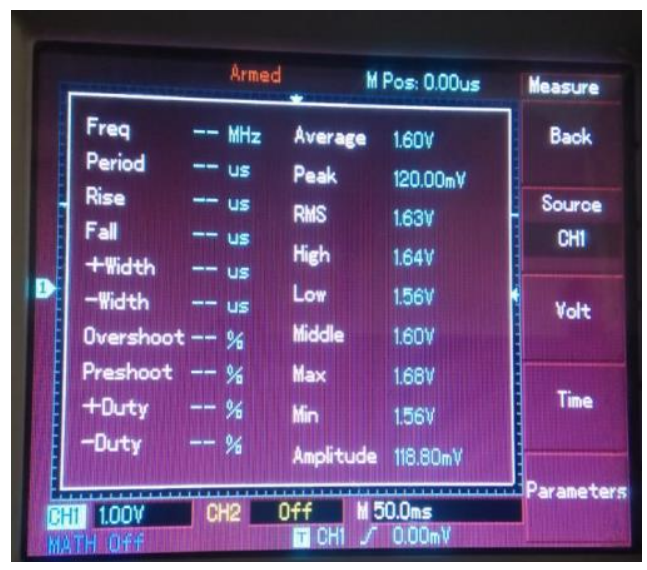

Fig 20: Thumb Flat Resistance Parameters

Figure 20 represents the voltage output at 0 degree angle and we took the average value of voltage which is $1.60 \mathrm{v}$ for thumb.

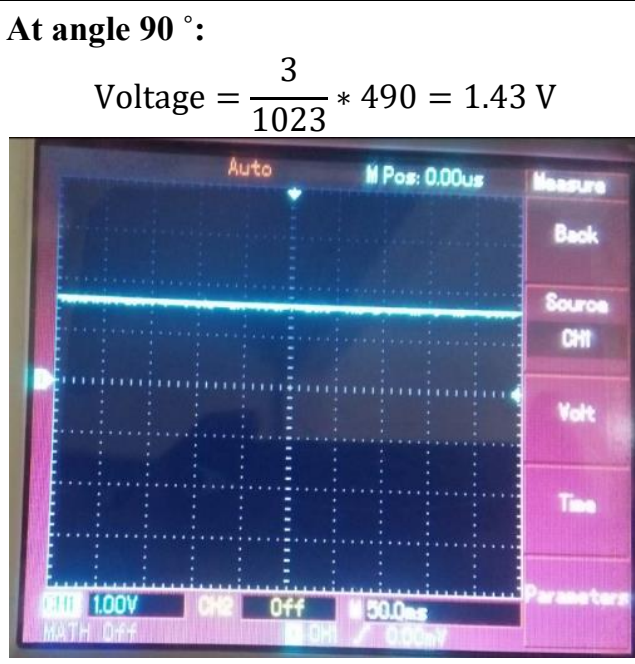

Fig 21: Thumb Bent Resistance Scope View

Figure 21 along $\mathrm{x}$-axix time is represented and along y-axix voltage is represented.

One block along $\mathrm{x}$-axix represents $1 \mathrm{v}$ and along y-axix one block shows $50 \mathrm{~ms}$ time period.

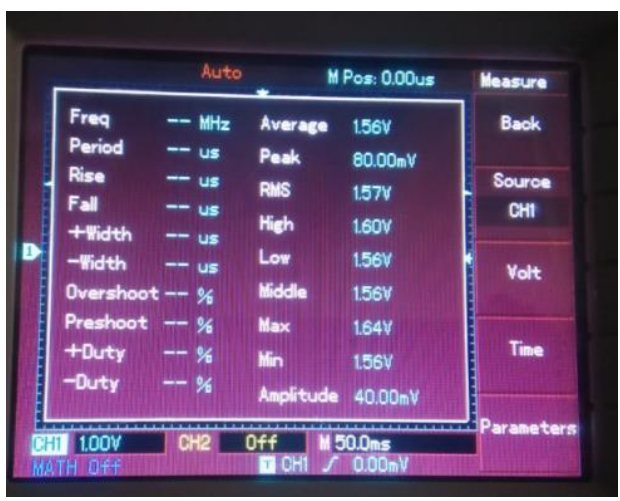

Fig 22: Thumb Bent Resistance Parameter

Figure 22 represents the voltage output at 90 degree angle and we took the average value of voltage which is $1.56 \mathrm{v}$ for thumb. 
Hamayun Khan (et al.) A Global System for Mobile Communication based Resource Allocation technique to control Autonomous Robotic Glove for Spinal Cord Implant Paralysed Patients using Flex Sensors

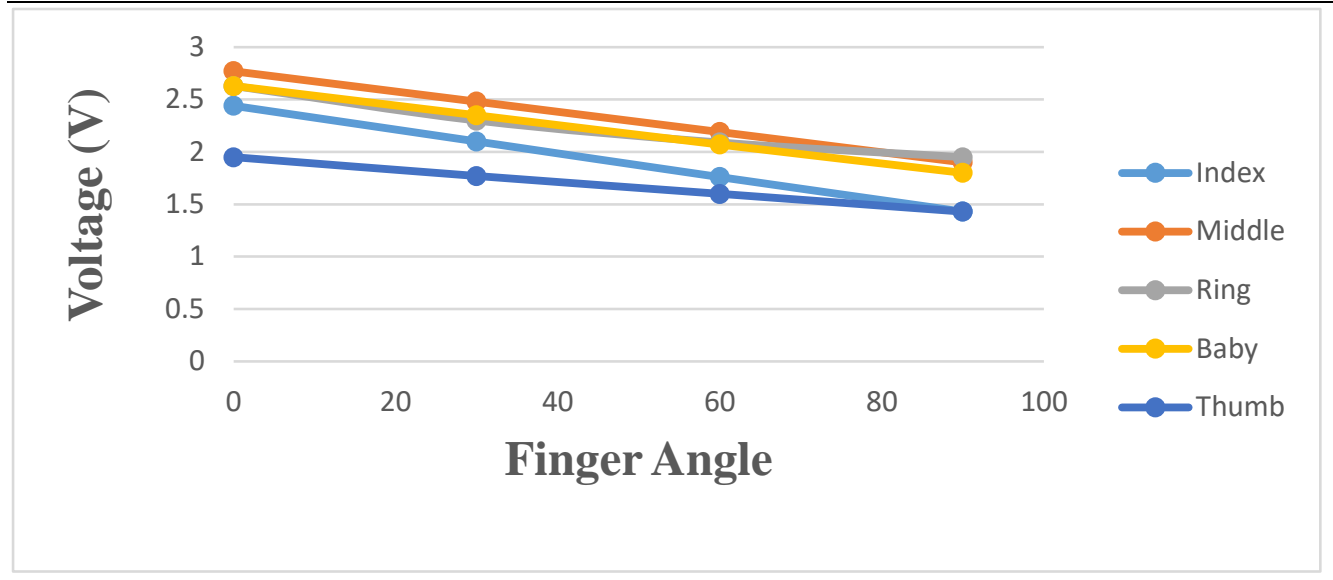

Fig 23: Variation of Voltage at Different Angles of Fingers

Above fig 23 describes the variation of voltage by twisting the all fingers including thumb with different angles. Along $\mathrm{x}$-axis angle is mentioned while along $\mathrm{y}$-axis voltage variation is mentioned. At angle 0 to 90 voltage level variation is mentioned with different colurs.

\section{Conclusion}

Many people in this world are paralyzed and disable and some of them are deaf and dumb. They are facing difficulties to communicate with others because the gesture language is not easily understood by the people. By using different types of sign gestures it is very difficult to communicate with them. but the our research is robotic glove for paralyzed patients or disable who are bed rest and can't move from one place to another and always need him/her for any kind of help so by using this system they will be able to control home appliance independently. The system is constructed to read hands of finger movement and translate this particular hands movement into the analog form. This method is very easy to contact with others, in which the user does not need any type of training and this is wireless technique in which the patient can communicate with others within hundred meters range. So in any case of danger e.g. earthquake or fires, if the patient is in hazard and need to get a help so he can just move his/her hands finger than the message will be send to a particular person.
Finally; this small system will effect in helping the humankind which is really a greatest act. The research is made from electronics devices which are placed on hand glove that is wearable, portable and can be easily used by the paralyzed patient and do some work without any kind of other helping. This system is more effective, efficient and low cast which serves the different types of disable person. This task shows the execution of a basic hand glove, to help the physically tested or confined to bed patients. The glove when worn can be utilized to work electrical devices, by the straight forward flexing of the fingers. The activity is determined by the fingers curved. It is very easy to construct. Its range is limited because of using HC-11. The range can be expanded by utilizing Zigbee or $\mathrm{HC}-12$.

\section{References}

[1] Zimmerman, T. G. (1985). U.S. Patent No. 4,542,291. Washington, DC: U.S. Patent and Trademark Office.

[2] Maharjan, P., Bhatta, T., Salauddin, M., Rasel, M. S., Rahman, M. T., Rana, S. M. S., \& Park, J. Y. (2020). A human skininspired self-powered flex sensor with thermally embossed microstructured triboelectric layers for sign language interpretation. Nano Energy, 76, 105071..

[3] Aira Patrice R. Ong, Nilo T. Bugtai "Recent Developments of Robotic Exoskeletons for Hand Rehabilitation" De La Salle University, Manila, 
Hamayun Khan (et al.) A Global System for Mobile Communication based Resource Allocation technique to control Autonomous Robotic Glove for Spinal Cord Implant Paralysed Patients using Flex Sensors (pp. $13-23)$

Philippines. vol 4 ,ISSN 2449-3309,Issue March 7-9, 2016

[4] Abbas U1*, Shaikh MZ2, Panhwer A2, Turk SK1 and Kumar N1. ."Design and Implementation of the Advanced Wireless Tongue Drive" Journal of Bioengineering \& Biomedical Science ISSN: 2155-9538 JBBS an open access journal Volume 6 |Issue 2 |1000185 Published Apr 26, 2016

[5] Nikhita Praveen ,Naveen Karanth ,M S Megha "Sign language interpreter using a robotic glove" International conference on advance in electronics, computers and communication 2014 IEEE

[6] H. Khan, M. U. Hashmi, Z. Khan, R. Ahmad, and A. Saleem, "Performance Evaluation for Secure DES-Algorithm Based Authentication \& Counter Measures for Internet Mobile Host Protocol," IJCSNS Int. J. Comput. Sci. Netw. Secur. VOL.18 No.12, December 2018, vol. 18 , no. 12 , pp. 181-185, 2018.

[7] James Connolly, Joan Condell, Brendan O'Flynn, Javier Torres Sanchez, and Philip Gardiner."IMU Sensor-Based Electronic Goniometric Glove for Clinical Finger Movement Analysis" IEEE SENSORS JOURNAL, VOL. 18, NO. 3, FEBRUARY 1, 2018

[8] Abhinandan Das, Lavish Yadav, Mayank Singhal, Raman Sachan, Hemang Goyal, Keshav Taparia Raghav Gulati, Ankit Singh, Gaurav Trived."Robotic glove for Sign Language communications" Department of Electronics and Electrical Engineering Indian Institute of Technology Guwahati Guwahati, Assam 781039, Indial 16-18 Dec 2016 IEEE

[9] Tushar Chouhan, Ankit Panse, Anvesh Kumar Voona and S. M. Sameer. "Robotic glove with gesture recognition ability for the hearing and speech impaired" 2014 IEEE Global Humanitarian Technology Conference South Asia Satellite (GHTC-SAS) | September 26-27, 2014 | Trivandrum

[10] H. Khan, M. U. Hashmi, Z. Khan, and R. Ahmad, "Offline Earliest Deadline first Scheduling based Technique for Optimization of Energy using STORM in Homogeneous Multi- core Systems," IJCSNS Int. J. Comput. Sci. Netw. Secur. VOL.18 No.12, December 2018, vol. 18, no. 12, pp. 125-130, 2018.

[11] Izzeldin Ibrahim Mohamed Abdelaziz. "A Low-Cost Robotic glove for Hand Functions Evaluation" International Journal of Robotics and Automation
(IJRA) Vol. 3, No. 1, March 2014, ISSN: 2089-4856

[12] Yeasin Ar Rahman, Mohammed Moshiul Hoque, Khalid Ibn Zinnah, Imam Mohammad Bokhary. "Helping-Hand: A Data Glove Technology for Rehabilitation of Monoplegia Patients" The 9th International Forum on Strategic Technology (IFOST), October 21-23, 2014, Cox's Bazar, Bangladesh 978-14799-6062-0/14/2014 IEEE

[13] Khan, M. Y. A. (2020). A high state of modular transistor on a $105 \mathrm{~kW}$ HVPS for X-rays tomography Applications. Sukkur IBA Journal of Emerging Technologies, 2(2), 1-6.

[14] Khan, H., Hashmi, M. U., Khan, Z., \& Ahmad, R. (2018). Offline Earliest Deadline first Scheduling based Technique for Optimization of Energy using STORM in Homogeneous Multicore Systems. IJCSNS Int. J. Comput. Sci. Netw. Secur, 18(12), 125-130. 